Nota clínica

\title{
Infarto agudo de miocardio inferior enmascarando el síndrome de la onda J. A propósito de cuatro observaciones
}

\author{
J. ORTEGA CARNICER
}

Servicio de Medicina Intensiva. Hospital General de Ciudad Real.

\begin{abstract}
El síndrome de la onda J está caracterizado por una onda $\mathrm{J}$ prominente acompañada de una elevación del segmento ST en ausencia de una enfermedad cardíaca estructural, e incluye el síndrome benigno de la repolarización precoz y los síndromes arritmogénicos de Brugada y de la fibrilación ventricular idiopática. Aunque los síndromes coronarios agudos son una de las principales causas de desviación del segmento ST, no existen casos clínicos que describan específicamente los efectos moduladores de una corriente de lesión isquémica sobre las manifestaciones electrocardiográficas del síndrome de la onda J. Este artículo describe 4 casos de pacientes con un infarto agudo de miocardio con elevación inferior del ST que tuvieron una onda $J$ (o desplazamiento negativo del punto J) y depresión del segmento ST en las derivaciones precordiales derechas. Después, estas alteraciones precordiales del ECG desaparecieron y fueron progresivamente reemplazadas por ondas $J\left(R^{\prime}\right)$ prominentes y elevaciones anteriores del segmento ST sugerentes de la presencia de un síndrome de la onda J. En conclusión, el síndrome de la onda J puede estar oculto por un infarto agudo de miocardio inferior con depresión simultánea del segmento ST en las derivaciones precordiales derechas. En estas circunstancias, la detección precoz de una onda $\mathrm{J}$ (o depresión del punto $\mathrm{J}$ ) puede utilizarse como marcador electrocardiográfico del síndrome de Brugada o de la repolarización precoz.
\end{abstract}

Correspondencia: Dr. J. Ortega Carnicer.

Los Alisos, 10.

13002 Ciudad Real.

Correo electrónico: jortegacar@gmail.com

Manuscrito aceptado el 4-I-2007.
PALABRAS CLAVE: infarto agudo de miocardio, síndrome de Brugada, síndrome de la repolarización precoz, onda J.

\section{ACUTE INFERIOR MYOCARDIAL INFARCTION MASKING THE J WAVE SYNDROME. BASED ON FOUR OBSERVATIONS}

The $\mathbf{J}$ wave syndrome is characterized by a prominent $\mathbf{J}$ wave accompanied by $S T$-segment elevation in the absence of structural heart disease. It includes the benign early repolarization syndrome, the highly arrhythmogenic Brugada syndrome and idiopathic ventricular fibrillation. Although acute coronary syndromes are one of the leading causes of ST-segment deviation, no clinical reports that specifically describe the modulating effects of an ischemic injury current on the ECG manifestations of the $J$ wave syndrome have been found. This report describes four cases of patients with acute inferior ST-segment elevation myocardial infarction who had $\mathrm{J}$ wave (or negative deplacement of the $\mathrm{J}$ point) and ST-segment depression in the right precordial leads. Later, these precordial ECG alterations disappeared and were progressively replaced by prominent $\mathbf{J}\left(\mathbf{R}^{\prime}\right)$ waves and anterior ST-segment elevations, suggesting the presence of a $\mathrm{J}$ wave syndrome. In conclusion, the $\mathrm{J}$ wave syndrome may be obscured by an acute inferior myocardial infarction with concomitant ST-segment depression in the right precordial leads. In such circumstances, early detection of the $\mathbf{J}$ wave (or depressed $\mathbf{J}$ point) may be used as ECG marker of the early repolarization syndrome or Brugada syndrome.

KEY WORDS: acute myocardial infarction, Brugada syndrome, early repolarization syndrome, $J$ wave. 


\section{INTRODUCCIÓN}

El síndrome de la onda J (SOJ) está caracterizado por una onda $\mathbf{J}$ prominente acompañada de una elevación del segmento ST (E-ST) en ausencia de una enfermedad cardíaca estructural, e incluye el síndrome benigno de la repolarización precoz (SRP) y los síndromes arritmogénicos de Brugada (SB) y de la fibrilación ventricular idiopática (una variante del SB con E-ST inferior) ${ }^{1}$. Aunque el SRP puede causar una E-ST en las derivaciones precordiales derechas que simula un $\mathrm{SB}^{2-4}$, el electrocardiograma (ECG) permite normalmente diferenciar estos dos procesos. $\mathrm{La}$ E-ST en el SRP está usualmente localizada en las derivaciones V2-V4, su morfología muestra una concavidad superior con polaridad positiva de la onda $\mathrm{T}$ y se acompaña por una onda $\mathrm{J}^{4,5}$, mientras que el ECG del SB generalmente muestra una onda J prominente seguida por una E-ST de forma convexa (y ocasionalmente en silla de montar) que finaliza en una onda $\mathrm{T}$ negativa en las derivaciones V1-V2 y a veces en $\mathrm{V}^{3,6}$. Aunque los síndromes coronarios agudos son una de las principales causas de desviación del segmento ST, llama la atención que no existen casos clínicos que describan específicamente los efectos moduladores de una corriente de lesión isquémica sobre las manifestaciones electrocardiográficas del SOJ.

Se muestran 4 pacientes con un infarto agudo de miocardio (IAM) con E-ST inferior que tuvieron simultáneamente una onda J y una depresión del segmento ST (D-ST) en las derivaciones precordiales derechas. Posteriormente, estas manifestaciones del ECG fueron reemplazadas por ondas J marcadas y E-ST anterior sugestivas del SOJ. Todos los pacientes carecieron de historia de fibrilación ventricular, síncope o historia familiar de muerte súbita. Ningún enfermo tuvo manifestaciones de infarto ventricular derecho.

\section{DESCRIPCIÓN DE LOS CASOS}

\section{Caso 1}

Varón de 54 años que ingresa en el hospital a causa de un episodio de dolor torácico de una hora de duración. El ECG (fig. 1A) mostró ondas Q junto a E-ST en derivaciones inferiores y D-ST en derivaciones I, aVL y V4-V5. En derivaciones precordiales derechas pudo observarse también una onda $\mathrm{J}$ marcada seguida de una D-ST convexa y descendente que finalizaba en una onda $\mathrm{T}$ negativa. Con el diagnóstico de IAM inferior recibió tratamiento fibrinolítico con tenecteplasa. Una hora después el ECG (fig. 1B), registrado con ligero dolor precordial, reveló ausencia de E-ST inferior, una onda T negativa en aVL y una elevación del punto $\mathrm{J}>0,2 \mathrm{mV}$ con E-ST descendente $\mathrm{y}$ onda $\mathrm{T}$ negativa en derivaciones V1-V2, que sugerían un patrón electrocardiográfico de SB. Cinco horas más tarde el ECG (fig. 1C) mostró una onda J prominente con E-ST convexa y en silla de montar en derivaciones V1 y V2, respectivamente. También hubo una elevación del punto J asociada con una E-ST y onda $\mathrm{T}$ positiva en derivaciones V3-V4. Tres días después del ingreso el ECG (fig. 1D) reveló un patrón $\mathrm{qR}$ inferior junto con una onda $\mathrm{T}$ negativa en derivaciones III y aVF, una onda J manifiesta en V1-V2, una E-ST cóncava con onda T positiva en derivaciones V2-V3, y ausencia de onda $\mathrm{S}$ en las derivaciones izquierdas. Mientras estuvo ingresado no tuvo arritmias ventriculares. El valor máximo de la creatinquinasa y troponina I fueron $1.398 \mathrm{U} / 1$ y $23,2 \mathrm{ng} / \mathrm{ml}$, respectivamente. La arteriografía coronaria reveló una estenosis $(95 \%)$ proximal de la arteria coronaria derecha y se le colocó un stent. Tras ser dado de alta, 6 meses más tarde fue sometido a una prueba de provocación con flecainida $(2 \mathrm{mg} / \mathrm{kg}$ intravenosamente en 10 minutos) que fue negativa para poder hacer un diagnóstico de SB.

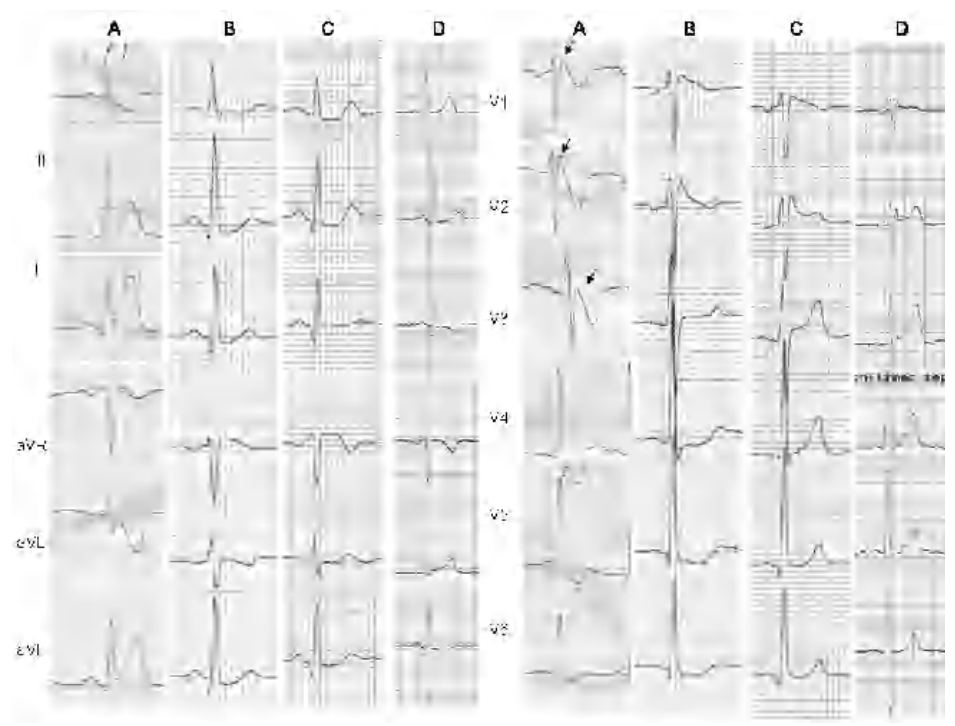

Figura 1. Electrocardiogramas del caso 1. Ver detalles en el texto. 


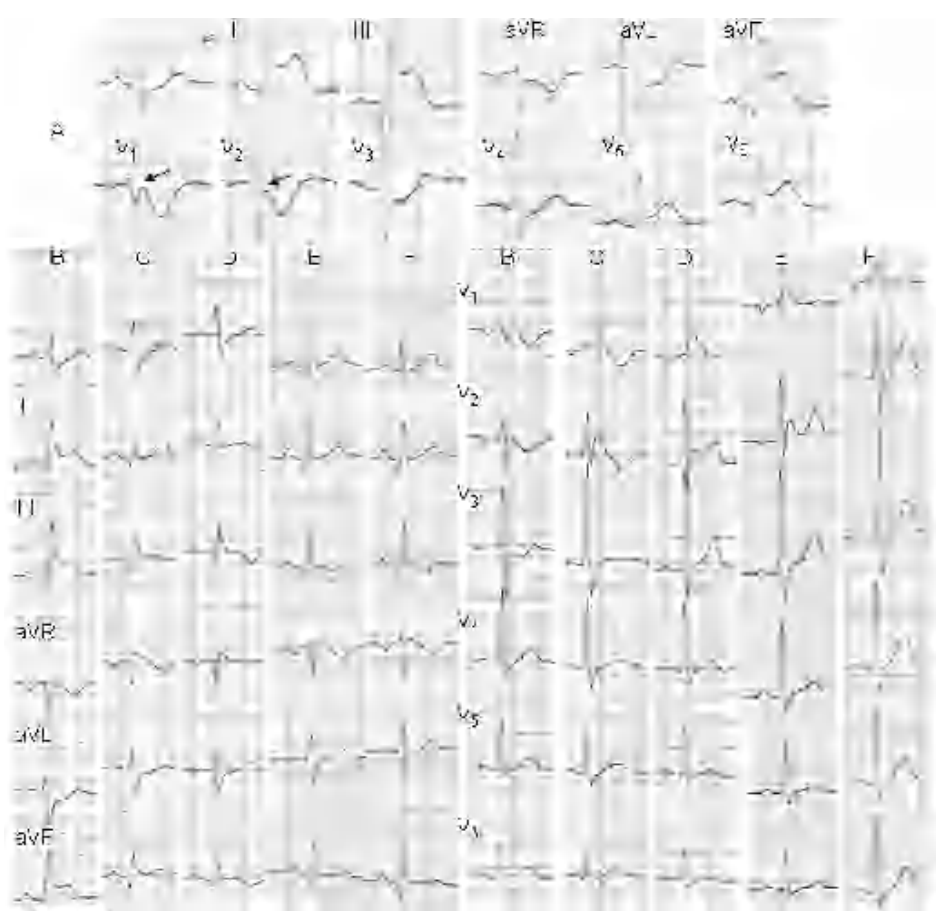

Figura 2. Electrocardiogramas del caso 2. Ver detalles en el texto.

\section{Caso 2}

Varón diabético de 68 años que acude al hospital por dolor retroesternal de tres horas de duración. El ECG (fig. 2A) mostró un patrón qR y E-ST en las derivaciones inferiores y una D-ST recíproca en I y aVL. También hubo un desplazamiento negativo del punto J seguido de una D-ST descendente en las derivaciones V1-V2 y horizontal en V3. Se diagnosticó de IAM inferoposterior con extensión lateral y recibió tratamiento fibrinolítico con tenecteplasa. Dos horas después del ingreso el ECG (fig. 2B) reveló un patrón qR y una menor E-ST en las derivaciones inferiores, una D-ST recíproca en I y aVL, una onda $\mathbf{J}$ seguida de una D-ST descendente y una onda T negativa en las derivaciones V1-V2 y ausencia de onda $\mathrm{S}$ en V5-V6. Doce horas después del ingreso, el ECG (fig. 2C) mostró un patrón qR con mínima E-ST en las derivaciones inferiores, una onda $\mathrm{R}$ alta en V2$\mathrm{V} 3$, una onda S estrecha en V5-V6, una pequeña onda $\mathrm{R}$ en aVR, y una nueva onda J (R') gigante asociada con una E-ST convexa y una onda T negativa en V1-V2. Estos datos del ECG se interpretaron como infarto de miocardio inferoposterior asociado con bloqueo de rama derecha (BRD) incompleto y patrón tipo SB. Un día después del ingreso el ECG (fig. 2D) reveló un patrón rR' (J) junto con E-ST convexa y onda $\mathrm{T}$ aplanada en la derivación V1 y una onda R alta con E-ST en silla de montar en V2. Al tercer día de ingreso el ECG (fig. 2E) puso de manifiesto un patrón rsR' con onda $\mathrm{T}$ positiva en la derivación $\mathrm{V} 1$ y una onda R grande con E-ST en silla de montar en V2. Los valores máximos de la creatinquinasa y de la troponina I fueron $2.279 \mathrm{U} / 1 \mathrm{y} 14,6 \mathrm{ng} / \mathrm{ml}$, respectiva- mente. La telemetría no pudo demostrar la aparición de arritmias. Un ecocardiograma puso de manifiesto una acinesia inferoposterior. El paciente rechazó la realización de una prueba de flecainida y una arteriografía coronaria. A los dos años del alta el ECG (fig. 2F) muestra un patrón de infarto de miocardio inferior, un complejo rsR' en la derivación V1 y una onda $\mathrm{S}$ en las derivaciones laterales izquierdas, una relación $\mathrm{R} / \mathrm{S}$ ratio > 1 en V2-V3 y un punto J manifiesto con E-ST en V2.

\section{Caso 3}

Varón de 60 años que acude al hospital por dolor precordial de dos horas de duración. El ECG (fig. 3A) reveló una corriente de lesión subepicárdica compatible con un IAM inferior, una depresión pronunciada del punto J junto a una D-ST descendente en las derivaciones V1-V2 y una D-ST horizontal en V3 a V5. Se administró tratamiento fibrinolítico con tenecteplasa y lidocaína por taquicardias ventriculares extrasistólicas. Una hora y media después el ECG (fig. $3 \mathrm{~B})$, registrado sin dolor, reveló una onda $\mathrm{J}$ marcada en derivaciones V1-V2 y ausencia de desplazamiento del segmento ST. Un día después el ECG (fig. 3C) puso de manifiesto una inversión inferior de la onda $\mathrm{T}$, una onda $\mathrm{J}$ en las derivaciones V1-V2 y una E-ST cóncava con ondas $\mathrm{T}$ positivas en las derivaciones V2 a V4. La creatinquinasa ascendió hasta $1.143 \mathrm{U} / 1$ (con una fracción MB de 102). Un ecocardiograma reveló una acinesia inferobasal. La evolución clínica hospitalaria no tuvo incidencias significativas. Cuatro años después del alta el ECG (fig. 3D) evidencia una onda 


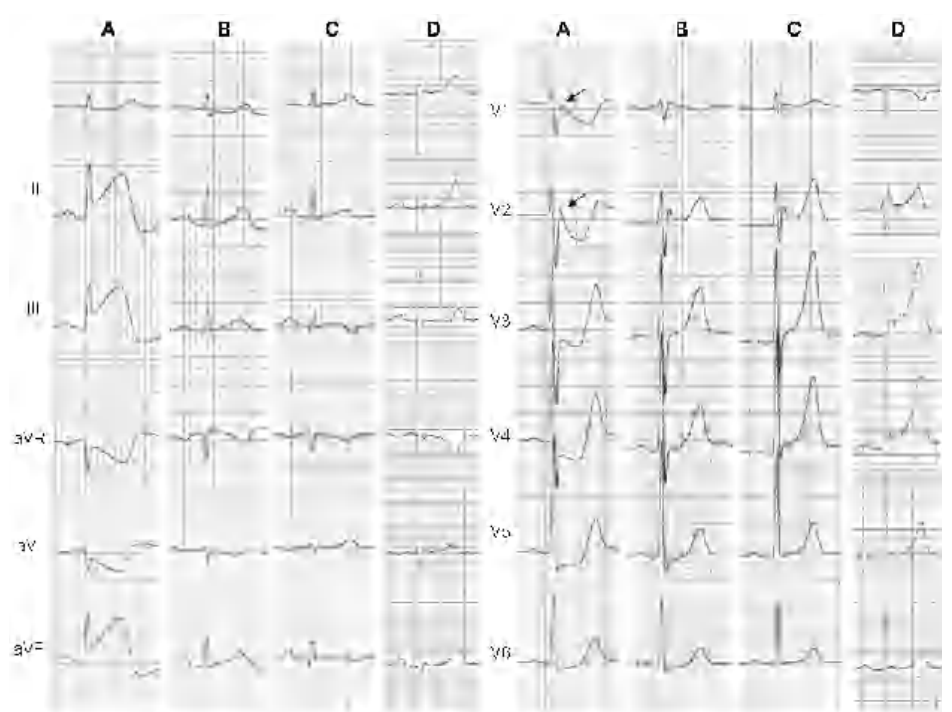

Figura 3. Electrocardiogramas del caso 3. Ver detalles en el texto.

$\mathrm{J}$ en las derivaciones inferiores y anteriores, y una E-ST cóncava con onda T positiva en las derivaciones V2 a V4 sugerente de un SRP.

\section{Caso 4}

Varón de 43 años que ingresa en el hospital por dolor precordial intenso de una hora de duración. El ECG (fig. 4A) mostró ligera E-ST inferior, onda J prominente en V1-V2 y D-ST marcada en derivaciones V1 a V5. Diagnosticado de IAM inferoposterior, el paciente recibió tratamiento trombolítico con reteplasa. Una hora más tarde el ECG (fig. 4B) reveló un BRD incompleto, una onda J (R') manifiesta en derivaciones V1-V2 y ausencia de desviaciones del segmento ST. También hubo ondas R altas afectando la parte inicial del complejo QRS y ondas T acuminadas en derivaciones V2-V3, que sugerían el diagnóstico de IAM posterior sobreañadido. A las 8 horas del ingreso el ECG (fig. 4C) puso de manifiesto una inversión de la onda $\mathrm{T}$ en derivaciones III y aVF, una onda $\mathrm{J}$ en V1-V2 y una E-ST cóncava con onda T positiva en derivaciones V1 a V4. Tres días después el ECG (fig. 4D) reveló una onda J, una E-ST cóncava y una onda T positiva de V2 a V5 que sugerían un SRP. La creatinquinasa ascendió hasta 4.999 U/l (con una fracción $\mathrm{MB}$ de 254). Un ecocardiograma reveló una acinesia inferobasal y apical. Una arteriografía coronaria puso de manifiesto una estenosis del $90 \%$ en el tercio medio de la arteria coronaria derecha, y de un $60 \%$ en la porción proximal de la arteria circunfleja. Tras la colocación de un stent en la arteria coronaria derecha, el paciente lleva tres años asintomático.

\section{DISCUSIÓN}

El hallazgo electrocardiográfico de una onda J asociada a una E-ST en las derivaciones precordiales de- rechas se ha observado en una variedad de situaciones experimentales y clínicas que incluyen el SRP ${ }^{1,4,5}$, el SB idiopático ${ }^{1-3,6}$ y las formas adquiridas del SB debidas a fármacos (antiarrítmicos, antianginosos y psicotropos), anormalidades electrolíticas (hiperpotasemia e hipercalcemia), isquemia aguda (infarto de miocardio ventricular derecho y angina vasoespástica), niveles de insulina, hipotermia o fiebre ${ }^{7,8}$.

Los ECG iniciales de estos pacientes con IAM inferior mostraron ondas $\mathrm{J}$ (o desplazamiento negativo del punto J) y D-ST en las derivaciones precordiales derechas. Luego, estos cambios precordiales del ECG desaparecieron y fueron progresivamente reemplazados por ondas J (R') prominentes y E-ST que sugerían la presencia de un SOJ. Los pacientes 1 (fig. 1A y 1B) y 2 (fig. 2C) mostraron el patrón electrocardiográfico de SB tipo 1, incluyendo una E-ST $\geq 2 \mathrm{~mm}$ con morfología convexa y onda $\mathrm{T}$ negativa en derivaciones $\mathrm{V} 1$ y V2, mientras que los ECG de los pacientes 3 (fig. 3D) y 4 (fig. 4D) revelaron una onda J y una E-ST con concavidad superior, y onda $\mathrm{T}$ positiva en derivaciones V2 a V4-5 característicos del SRP.

Aunque esta secuencia electrocardiográfica no ha sido descrita con anterioridad, la combinación de un IAM y el SOJ es interesante en dos aspectos. En primer lugar el SOJ estuvo oculto por un IAM inferior con D-ST simultánea en las derivaciones precordiales. En estas circunstancias una corriente de lesión subepicárdica en la pared posterior del ventrículo izquierdo («imagen en espejo») o una lesión subendocárdica en la pared anterior cancelaron la E-ST anterior característica del SOJ. Realizando una revisión de la literatura centrada sobre pacientes con SB y angina variante con E-ST inferior o lateral, he encontrado varios casos publicados por Imazio et $\mathrm{al}^{9}$, Chinushi et $\mathrm{al}^{10}$, Sasaki et $\mathrm{al}^{11}$ y Marill y Ellinor ${ }^{12}$ que mostraban un enmascaramiento del SB por atenuación o depresión de la E-ST anterior durante un vasoespasmo de la arteria coronaria derecha o circunfleja. 


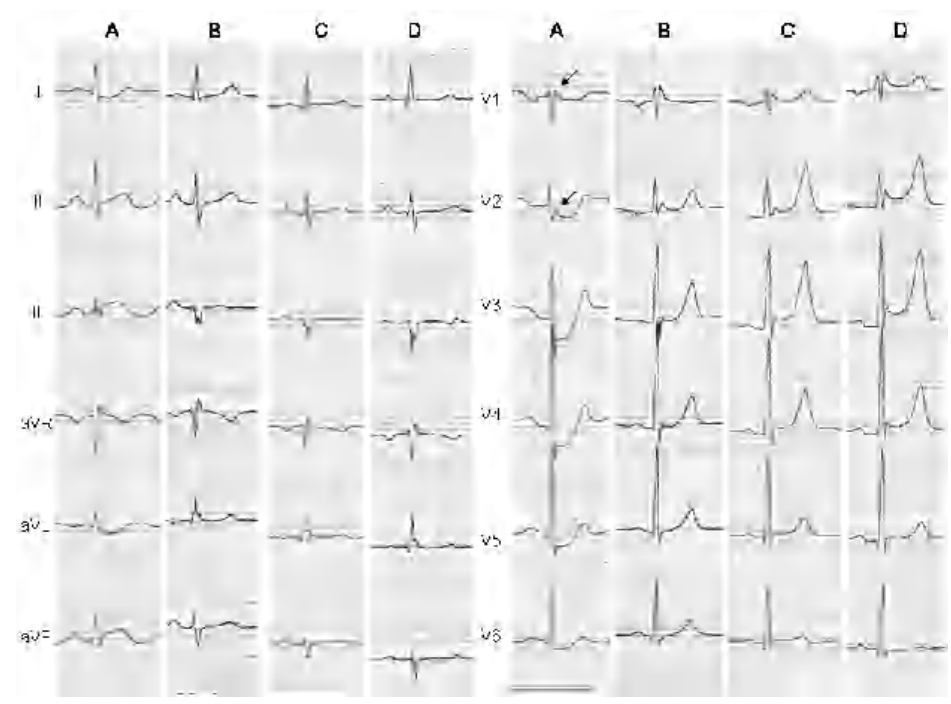

Figura 4. Electrocardiogramas del caso 4. Ver detalles en el texto.

Recientemente, Sassone et $\mathrm{al}^{13}$ describieron un paciente con patrón electrocardiográfico típico de SB, en quien la administración de ajmalina provocó la aparición de ondas J y E-ST en la derivaciones inferiores, mientras las anormalidades basales del ECG en las derivaciones V1 a V3 se normalizaban. Luego, tras la infusión de isoproterenol, el patrón electrocardiográfico del SB reapareció. Estos efectos opuestos de la ajmalina y el isoproterenol sobre el ECG podrían ser explicados también por una manifestación recíproca de la E-ST en la pared inferior, que anuló el efecto sobre la pared anterior. En segundo lugar la detección precoz de una onda $\mathrm{J}$ (o el desplazamiento negativo del punto J) en las derivaciones V1 y V2 puede usarse como un marcador electrocardiográfico de la presencia subyacente de un SOJ. Así, Marill y Ellinor $^{12}$ refirieron un paciente con SB que tuvo una parada cardíaca mientras dormía, y su ECG de ingreso mostró grandes semejanzas con los casos aquí descritos. Aunque no mencionado por los autores, el ECG inicial reveló una sutil onda $J$ asociada con una ligera D-ST en las derivaciones V1 y V2 durante un episodio de isquemia miocárdica transmural inferolateral. Siete horas más tarde estos cambios del ECG fueron sustituidos por una onda $\mathrm{J}$ marcada, seguida de una E-ST descendente en las derivaciones precordiales derechas características del SB. De forma similar, el caso de un paciente con SB publicado por Guevara-Valdivia et al ${ }^{14}$ mostró una normalización del segmento ST en V2 durante una prueba de esfuerzo, pero un detallado análisis del trazado también reveló una onda J mínima y una ligera D-ST descendente que precedía al patrón electrocardiográfico del $\mathrm{SB}$, visible en su totalidad durante el período de recuperación del ejercicio.

Aunque el IAM o la isquemia en el tracto de salida del ventrículo derecho puede causar una E-ST simulando un $\mathrm{SB}^{15-17}$, este mecanismo no estuvo implicado en los casos presentados, pues la E-ST anterior apareció tras la normalización del ST en la cara inferior y luego permaneció crónicamente elevado a lo largo del tiempo. Un BRD completo también puede causar una repolarización ventricular anormal en las derivaciones precordiales derechas ${ }^{18}$; sin embargo, en estos casos las anormalidades del segmento ST fueron claramente diferentes a las vistas en los BRD completos no complicados.

La E-ST presente en el SOJ y en el IAM tiene un mecanismo de producción similar, que se basa en la pérdida o depresión de la cúpula del potencial de acción en el epicardio, pero no en el endocardio, lo que genera un gradiente de voltaje que lleva a la E-ST ${ }^{1,19-22}$. Por tanto, la coexistencia del SOJ y del IAM puede ser clínicamente importante, pues fármacos utilizados rutinariamente en la cardiopatía isquémica (nitratos, antagonistas del calcio o bloqueadores beta) pueden aumentar las corrientes iónicas hacia fuera (Ito o IKATP) o reducirlas hacia dentro (Ica o INa), lo cual podría aumentar la magnitud del gradiente del potencial de acción y ocasionar mayor E-ST y fibrilación ventricular en pacientes con el SB, isquemia miocárdica o IAM $^{8,23,24}$.

En conclusión, un IAM inferior con D-ST simultánea en las derivaciones precordiales derechas puede enmascarar un SOJ. En estas circunstancias la detección precoz de una onda $\mathbf{J}$ (o depresión del punto $\mathrm{J}$ ) puede ser utilizada como marcador electrocardiográfico de un SB o SRP subyacente.

\section{Declaración de conflicto de intereses}

El autor ha declarado no tener ningún conflicto de intereses.

\section{BIBLIOGRAFÍA}

1. Shu J, Zhu T, Yang L, Cui C, Yan GX. ST-segment elevation in the early repolarization syndrome, idiopathy ventricular fibrillation, and the Brugada syndrome: cellular and clinical linkage. J Electrocardiol. 2005;38:26-32. 
ORTEGA CARNICER J. INFARTO AGUDO DE MIOCARDIO INFERIOR ENMASCARANDO EL SÍNDROME DE LA ONDA J. A PROPÓSITO DE CUATRO OBSERVACIONES

2. Antzelevitch C, Brugada P, Borggrefe M, Brugada J, Brugada $\mathrm{R}$, Corrado D, et al. Brugada syndrome: report of the second consensus conference endorsed by the Heart Rhythm Society and the European Heart Rhythm Association. Circulation. 2005;111:659-70.

3. Gussak I, Antzelevitch C, Bjerregarard P, Towbin JA, Chaitman BR. The Brugada syndrome: Clinical, electrophysiological and genetic aspects. J Am Coll Cardiol. 1999;33:5-15.

4. Gussack I, Antzelevitch C. Early repolarization syndrome: clinical characteristic and possible cellular and ionic mechanisms. J Electrocardiol. 2000;33:299-309.

5. Mehta M, Jain AC, Mehta A. Early repolarization. Clin Cardiol. 1999;22:59-65.

6. Brugada P, Brugada J. Right bundle branch block, persistent ST segment elevation and sudden cardiac death: a distinct clinical and electrocardiographic syndrome. J Am Coll Cardiol. 1992;20:1391-6.

7. Littmann L, Monroe MH, Kerns WP, Svenson RH, Gallagher JJ. Brugada syndrome and "Brugada sign": Clinical spectrum with a guide for the clinician. Am Heart J. 2003;145:768-78.

8. Shimizu W. Adquired forms of Brugada syndrome. En: Antzelevitch C, Brugada P, Brugada J, Brugada R, editors. The Brugada syndrome: from bench to bed side. 1th ed. Oxford: Blackwell-Futura; 2004. p. 78.

9. Imazio M, Ghisio A, Coda L, Tidu M, Belli R, Trinchero R, et al. Brugada syndrome: a case report of an unusual association with vasospastic angina and coronary myocardial bridging. Pacing Clin Electrophysiol. 2002;25:513-5.

10. Chinushi M, Furushima H, Tanabe $\mathrm{Y}$, Washizuka T, Aizawaz Y. Similarities between Brugada syndrome and ischemiainduced ST-segment elevation. Clinical correlation and synergy. J Electrocardiol. 2005;38:18-21.

11. Sasaki T, Niwano S, Kitano Y, Izumi T. Two cases of Brugada syndrome associated with spontaneous clinical episodes of coronary vasospasm. Intern Med. 2006;45:77-80.

12. Marill KA, Ellinor PT. Case 37-2005: A 35-year-old man with cardiac arrest while sleeping. N Engl J Med. 2005;353:2492-501.

13. Guevara-Valdivia ME, Iturralde Torres $P$, Micheli A, Colin Lizalde L, Medeiros Domingo A, González-Hermosillo JA. Elec- trocardiographic changes during stress test in a patient with "Brugada syndrome". Arch Cardiol Mex. 2001;71:66-72.

14. Sassone B, Saccà S, Donateo M. Paradoxical effect of ajmaline in a patient with Brugada syndrome. Europace. 2006;8: 251-4.

15. Kataoka H, Kanzaki K, Mikuriya Y. Massive ST-segment elevation in precordial and inferior leads in right ventricular myocardial infarction. J Electrocardiol. 1988;21:115-20.

16. Nakazato Y, Kurata T, Yamaguchi H. ST segment elevation in the precordial leads mimicking Brugada syndrome. Heart. 2000;83:216.

17. Ortega-Carnicer J, Martín-Rodríguez C, Portilla-Botelho M. Right ventricular infarction simulating the Brugada syndrome. Resuscitation. 2006;69:348-50.

18. Márquez MF, Bisteni A, Medrano G, De Micheli A, Guevara M, Iturralde P, et al. Dynamic electrocardiographic changes after aborted sudden death in a patient with Brugada syndrome and rate-dependent right bundle branch block. J Electrocardiol. 2005;38:256-9.

19. Di Diego JM, Antzelevitch C. Cellular basis for ST-segment changes observed during ischemia. J Electrocardiol 2003;36 Supl: 1-5.

20. Yan GX, Lankipalli RS, Burke JF, Musco S, Kowey PR. Ventricular repolarization components on the electrocardiogram. Cellular basis and clinical significance. J Am Coll Cardiol. 2003; 42:4019.

21. Di Diego JM, Fish JM, Antzelevitch C. Brugada syndrome and ischemia-induced ST-segment elevation. Similarities and differences. J Electrocardiol. 2005;38:14-7.

22. Yan GX, Antzelevitch C. Cellular basis for the electrocardiographic J wave. Circulation. 1996;93:372-9.

23. Matsuo K, Shimizu W, Kurita T, Inagachi M, Aihara N, Kamakura S. Dynamic changes of 12-lead electrocardiograms in a patient with Brugada syndrome. J Cardiovasc Electrophysiol. 1998;9:508-12.

24. Aouate P, Clerc J, Viard P, Seout J. Propranolol intoxication revealing a Brugada syndrome. J Cardiovasc Electrophysiol. 2005; 16:348-51. 